C. T. Chong

Nagoya Math. J.

Vol. 115 (1989), 165-183

\title{
MAXIMAL SETS AND FRAGMENTS OF PEANO ARITHMETIC
}

\section{C.T. CHONG}

This work is inspired by the recent paper of Mytilinaios and Slaman [9] on the infinite injury priority method. It may be considered to fall within the general program of the study of reverse recursion theory: What axioms of Peano arithmetic are required or sufficient to prove theorems in recursion theory? Previous contributions to this program, especially with respect to the finite and infinite injury priority methods, can be found in the works of Groszek and Mytilinaios [4], Groszek and Slaman [5], Mytilinaios [8], Slaman and Woodin [10]. Results of [4] and [9], for example, together pinpoint the existence of an incomplete, nonlow r.e. degree to be provable only within some fragment of Peano arithmetic at least as strong as $P^{-}+I \Sigma_{2}$. Indeed an abstract principle on infinite strategies, such as that on the construction of an incomplete high r.e. degree, was introduced in [4] and shown to be equivalent to $\Sigma_{2}$ induction over the base theory $P^{-}+I \Sigma_{0}$ of Peano arithmetic.

In this paper we study the problem of constructing maximal sets within fragments of Peano arithmetic, using ideas and techniques from $\alpha$ recursion theory. We show firstly that $P^{-}+B \Sigma_{2}$ does not prove the existence of such sets. The idea for this independence result comes from [9] which adapted the method of Shore [11], in his analysis of the jump of $\boldsymbol{\aleph}_{\omega}^{L}$-r.e. degrees, to produce a model $\mathscr{I}$ of $B \Sigma_{2}$ with no incomplete high r.e. degree. Here we investigate the same model and show that maximal sets do not exist in $\mathscr{I}$. This implies that there is no finite injury construction of a maximal set. Next we construct a maximal set in every model of $P^{-}+I \Sigma_{3}$. This shows that the existence of maximal sets is a theorem of this theory. We then prove that the statement on existence is consistent with the weaker theory $\mathrm{P}^{-}+\neg B \Sigma_{3}+I \Sigma_{2}{ }^{(*)}$ Finally, we show that there is a model of $P^{-}+\neg B \Sigma_{1}+I \Sigma_{0}$ (hence of $\neg B \Sigma_{2}$ ) with a maximal set. These results together show that the sentence

Received September 18, 1987.

(*) Cf. Note added in proof. 
asserting the existence of maximal sets is strictly weaker than $I \Sigma_{3}$ over the base theory $P^{-}+I \Sigma_{2}$, and does not imply nor is it implied by either $B \Sigma_{2}$ or $I \Sigma_{1}$. The appropriate references here for the background in $\alpha$ recursion theory are Lerman and Simpson [7], Chong and Lerman [2], and Chong [1].

We begin by recalling some basic notions in Peano arithmetic. Let $\mathscr{L}$ be the language of Peano arithmetic. This consists of the binary operations ' (succesor), + (addition) and - (multiplication) and the constant symbol 0 . We will use $t^{\prime}$ and $t+1$ interchangeably to denote successors. The relation $x<y$ is defined as $(\exists t)\left(x+t^{\prime}=y\right)$, while $x \leq y$ is a short form for $x<y \vee x=y$. Bounded quantifiers are quantifiers of the form $(\exists x \leq y)$ or $(\forall x \leq y)$. Define a formula to be $\Sigma_{0}$ or $\Pi_{0}$ if it has only bounded quantifiers. A formula $\varphi$ is $\Sigma_{n+1}$ if it is of the form $(\exists \vec{x}) \psi$ where $\psi$ is $\Pi_{n}$, while $\varphi$ is $\Pi_{n+1}$ if it is of the form $(\forall \vec{x}) \psi$ where $\psi$ is $\Sigma_{n}$, $((\exists \vec{x})$ and $(\forall \vec{x})$ denote respectively a block of existential quantifiers and a block of universal quantifiers). We let $P^{-}$denote the usual axioms of Peano arithmetic excluding mathematidal induction. The full mathematical induction can be split into levels of varying complexity as follows. For $n<\omega$, let $\Sigma_{n}$ induction (abbreviated $I \Sigma_{n}$ ) denote the following schema:

$$
(\forall \vec{y})\left[\left(\theta(\vec{y}, 0) \text { and }(\forall x)\left(\theta(\vec{y}, x) \longrightarrow \theta\left(\vec{y}, x^{\prime}\right)\right)\right) \longrightarrow(\forall x)(\theta(\vec{y}, x))\right],
$$

where $\theta$ is a formula. Clearly Peano arithmetic, which we denote by $P$, is $P^{-}$together with $I \Sigma_{n}$ for each $n$. Within each model $\mathscr{M}$ of $P^{-}+I \Sigma_{0}$, one can code finite sequences of elements of $\mathscr{M}$ by a single element. This is an important property that is needed in many constructions of r.e. sets.

A notion related to $I \Sigma_{n}$ is $L \Sigma_{n}$ (the principle of least $\Sigma_{n}$ element), which is syntactically stated as follows:

$$
(\forall \vec{w})[(\exists x) \theta(\vec{w}, x) \longrightarrow(\exists x)(\theta(\vec{w}, x) \text { and } \quad(\forall z<x) \neg \theta(\vec{w}, z))],
$$

where $\theta$ is a relation.

Sandwiched between $P^{-}+I \Sigma_{n-1}$ and $P^{-}+I \Sigma_{n}$ is the theory $P^{-}+$ $B \Sigma_{n}$, where $B \Sigma_{n}$ is the schema of $\Sigma_{n}$ collection: If $\theta$ is $\Sigma_{n}$ then

$$
(\forall \vec{w})(\forall y)[(\forall x<y)(\exists t) \theta(\vec{w}, t, x) \longrightarrow(\exists z)(\forall x<y)(\exists t<z) \theta(\vec{w}, t, x)] .
$$

Let $I \Pi_{n}, B \Pi_{n}$ and $L \Pi_{n}$ be defined similar to the corresponding ones for $\Sigma_{n}$ above, except that $\theta$ is now a $\Pi_{n}$ formula. The following 
result is due to Kirby and Paris [6]:

Proposition 1. Let $n<\omega$. Assume $P^{-}+I \Sigma_{0}$. Then

(a) $B \Sigma_{n+1}$ is equivalent to $B \Pi_{n}$;

(b) $I \Sigma_{n}, I \Pi_{n}, L \Sigma_{n}$ and $L \Pi_{n}$ are all equivalent;

(c) $I \Sigma_{n+1}$ implies $B \Sigma_{n+1}$, and $B \Sigma_{n+1}$ implies $I \Sigma_{n}$.

The standard model of Peano arithmetic will be denoted $\mathscr{N}$. Given $\mathscr{M}$ a model of some fragment of $P$, we define an initial segment $\mathscr{I}$ to be a nonempty subset of $\mathscr{M}$ closed under the successor operation ' and closed downward in $\mathscr{M}$ (i.e. if $b \in \mathscr{I}$ and $a<b$ then $a \in \mathscr{I}$ ). If $\mathscr{I}$ is proper, then it is called a proper initial segment of $\mathscr{M}$. Given $a \in \mathscr{M}$, we also denote by $a$ the set of all predecessors of $a$. The overspill lemma states that proper initial segments are not definable:

Proposition 2. Let $n<\omega$ and let $\mathscr{M}$ be a model of $P^{-}+I \Sigma_{n}$. If $\mathscr{I}$ is a proper initial segment of $\mathscr{M}$, then it is not $\Sigma_{n}$ definable with parameters over $\mathscr{M}$.

Proof. Suppose that $\mathscr{I}=\{a \mid \mathscr{M} \vDash \theta(a, \vec{b})\}$, where $\theta$ is $\Sigma_{n}$. Since $\mathscr{I}$ is a proper initial segment, $\mathscr{M} \backslash \mathscr{I}$ is a nonempty $\Pi_{n}$ set over $\mathscr{M}$. By hypothesis and by Proposition $1(b)$ this set has a least element $a_{0}$. Since $\mathscr{I} \neq \emptyset$, we see that $a_{0} \neq 0$. Then $a_{0}$ has an immediate predecessor which must belong to $\mathscr{I}$. But then $\mathscr{I}$ is not closed under the successor operation, a contradiction.

Definition. Let $\mathscr{M}$ be a model of $P^{-}+I \Sigma_{n}$, for some $n<\omega$. A bounded set $A \subset \mathscr{M}$ is $\mathscr{M}$-finite if there is an $a \in \mathscr{M}$, a formula $\varphi$ and elements $\vec{b} \in a$ such that for all $x$,

$$
x \in A \longleftrightarrow a \vDash \varphi(\vec{b}, x) .
$$

Using the pairing function (within $P^{-}+I \Sigma_{0}$ ), it is possible to code $\mathscr{M}$-finite sets by single elements of $\mathscr{M}$. Thus $\mathscr{M}$-finite sets are precisely those which are coded in $\mathscr{M}$. If $\mathscr{M}=\mathscr{N}$, then the $\mathscr{M}$-finite sets are just the finite sets of natural numbers. There exist, however, models $\mathscr{M}$ of $P^{-}+I \Sigma_{n}$ with many more $\mathscr{M}$-finite sets, as the following example constructed in [9] shows:

Lemma 1. There is a model $\mathscr{M}_{0}$ of Peano arithmetic such that for each real $E$, there is an $\mathscr{M}_{0}$-finite set $K$ such that $K \cap \mathscr{N}=E$. 
Let $\mathscr{M} \vDash P^{-}+I \Sigma_{n}$ for some $n$. The following notions apply to $\mathscr{M}$ : A set $A \subset \mathscr{M}$ is recursively enumerable (r.e.) if it is $\Sigma_{1}$ definable (with parameters) over $\mathscr{M} . \quad A$ is recursive if both $A$ and $\mathscr{M} \backslash A$ are r.e. (hence $\Delta_{1}$ over $\mathscr{M}$ ). A function $f: \mathscr{M} \rightarrow \mathscr{M}$ is (partial) recursive if its graph is r.e. It is total recursive if its domain is all of $\mathscr{M}$. There is a simultaneous recursive enumeration of all $\mathscr{M}$-finite sets and r.e. sets in $\mathscr{M}$. We let $K_{e}$ be the eth $\mathscr{M}$-finite set and let $W_{e}$ be the eth r.e. set. Denote by $\emptyset^{\prime}$ the set $\left\{(a, e) \mid a \in W_{e}\right\}$. This is a complete r.e. set. Finally $A$ is recursive in $B$ if there exist r.e. sets $W_{d}$ and $W_{e}$ such that for all $x$,

$$
\begin{aligned}
& x \in A \longleftrightarrow(\exists n)(\exists m)\left[(x, n, m, 1) \in W_{d} \text { and } K_{n} \subseteq B \text { and } K_{m} \subseteq \bar{B}\right] \\
& x \notin A \longleftrightarrow(\exists n)(\exists m)\left[(x, m, n, 1) \in W_{e} \text { and } K_{n} \subseteq B \text { and } K_{m} \subseteq \bar{B}\right] .
\end{aligned}
$$

Just as in $\alpha$ recursion theory, this relation is not necessarily transitive. To obtain transitivity, one replaces $x \in A$ by $K \subset A$ and $x \notin A$ by $K \not \subset A$, where $K$ is $\mathscr{M}$-finite. Such a modification is not needed here.

\section{Non-existence of maximal sets}

The following was proved by Mytilinaios and Slaman [9]:

Lemma 2. Let $\mathscr{M}_{0}$ be as given in Lemma 1. There is a proper initial segment $\mathscr{I}$ of $\mathscr{M}_{0}$ with the following properties:

(a) $\mathscr{I} \vDash P^{-}+B \Sigma_{2}$;

(b) $\mathscr{I}$ is a $\Sigma_{1}$ elementary substructure of $\mathscr{M}_{0}$; indeed there is an increasing sequence $\left\{a_{n}\right\}_{n<\omega} \subset \mathscr{I}$ such that $\mathscr{I}_{n+1}=\left\{x \mid x<a_{n+1}\right\}$ is the downward closure of the hull of $\mathscr{I}_{n}$ in $\mathscr{M}_{0}$ with respect to the first $n+1 \Sigma_{1}$ functions. Furthermore $\mathscr{I}=\cup \mathscr{I}_{n}$;

(c) There is a function $f: \mathscr{N} \rightarrow \mathscr{I}$ such that for each $n, f(n)=a_{n}$ (where $\left\{a_{n}\right\}_{n<\omega}$ is given in (b)), and such that $f$ is recursive in $\emptyset^{\prime}$.

The notion of regularity was first introduced in the context of $\alpha$ recursion theory. Its relevance to classical recursion theory, in connection with the problem of the existence of incomplete high sets, was noted in [9]. The study of the existence of maximal sets which we take up next also hinges on this notion. We first introduce a notation: For each r.e. set $W_{e}$, let $W_{e}^{s}$ be the set of elements enumerated into $W_{e}$ by the end of stage $s$.

Definition. Let $\mathscr{M}$ be a model of $P^{-}+I \Sigma_{n} . A$ set $A \subset \mathscr{M}$ is regular if $A \mid m$ is $\mathscr{M}$-finite for each $m \in \mathscr{M}$. 
Lemma 3. Let $\mathscr{M}$ be a model of $P^{-}+B \Sigma_{2}$. If $A \subset \mathscr{M}$ is r.e, then for each $m$, there is an such that

$$
(\forall x<m)(\exists t)\left[x \in A^{t} \longleftrightarrow x \in A^{s}\right] .
$$

In particular, $A$ is regular.

Proof. Let $m$ be given. Then

$$
(\forall x<m)(\exists s)\left[\left(x \in A^{s}\right) \vee(\forall t)\left(x \notin A^{t}\right)\right] .
$$

Since $\mathscr{M}$ is a model of $B \Sigma_{2}$, there is an $s$ such that

$$
(\forall x<m)\left[(\exists u<s)\left(x \in A^{u}\right) \vee(\forall t)\left(x \notin A^{t}\right)\right] .
$$

Formula (1) shows that $A$ is regular.

Remark. A result attributed to $\mathrm{H}$. Freidman states that the hypothesis in Lemma 3 may be weakened to $P^{-}+I \Sigma_{1}$.

Lemma 4. Let $\mathscr{I}$ be as given in Lemma 2. Let $f: \mathscr{N} \rightarrow \mathscr{I}$ be a cofinal map recursive in $\emptyset^{\prime}$. Then there is a partial recursive function $h: \mathscr{I} \times \mathscr{I}$ $\rightarrow \mathscr{I}$ such that for each $n \in \mathscr{N}$, for each $s, h(s, n)$ is defined, and that

$$
(\forall i \in \mathscr{N})[(\exists s)(\forall t \geq s) h(t, i)=f(i)] .
$$

We say that $h$ is a recursive approximation of $f$ and write $\lim _{s} h(s, i)=f(i)$ for $i \in \mathscr{N}$.

Proof. Let $e$ be chosen such that

$$
(i, z) \in f \longleftrightarrow(\exists n)(\exists m)\left[((i, z), n, m, 1) \in W_{e} \text { and } K_{n} \subset \emptyset^{\prime} \text { and } K_{m} \subset \emptyset^{\prime}\right] \text {. }
$$

By Lemma $3, \emptyset^{\prime}$ is regular, so that for all $a \in \mathscr{I}, \emptyset^{\prime} \cap a$ is $\mathscr{M}_{0}$-finite, hence $\mathscr{I}$-finite since $\mathscr{I}$ is a $\Sigma_{1}$ elementary substructure of $\mathscr{M}_{0}$. Fix $(i, z)$, and let $K_{n}$ and $K_{m}$ be $\mathscr{I}$-finite sets verifying $(i, z) \in f$. Let $g$ be total recursive such that $\emptyset^{\prime}=\operatorname{Range}(g)$. Since each $\mathscr{I}$-finite set is bounded, there is a stage $u$ in the recursive enumeration of $\emptyset^{\prime}$ such that for all $x$,

$$
\begin{aligned}
& x \in K_{n} \longrightarrow(\exists w<u)[g(w)=x], \text { and } \\
& x \in K_{m} \longrightarrow(\forall w)[g(w) \neq x] .
\end{aligned}
$$

Define $h(t, i)$ to be the least $z$ such that $((i, z), n, m, 1) \in W_{e}$ at some stage $u>t$, and such that there exist $K_{n}$ and $K_{m}$ satisfying (2) and

$$
x \in K_{m} \longrightarrow(\forall w<u)[g(w) \neq x] .
$$


Notice that for $i \in \mathscr{N}$, a $z$ to satisfy $((i, z), n, m, 1) \in W_{e}$ and (2), (4) at $u$ always exists since $f$ is total on $\mathscr{N}$. From the fact that $\mathscr{I}$ is a model of $B \Sigma_{2}$, hence of $I \Sigma_{1}$ (Proposition $1(c)$ ), and the fact that $I \Sigma_{1}$ is equivalent to $L \Sigma_{1}$ (Proposition $1((b)$ ), we see that the least $z$ required to define $h(t, i)$ can be found recursively for each $i \in \mathscr{N}$.

It is now not difficult to verify that $h$ is a recursive approximation of $f$.

Observe that if $i \notin \mathscr{N}$, then although $h(s, i)$ may be defined for many stages $s$, there is no $\lim _{s} h(s, i)$.

Definition. Let $\mathscr{M}$ be a model of $P^{-}+I \Sigma_{n}$. An r.e. set $M \subset \mathscr{M}$ is maximal if:

(a) For all $A \supseteq M$ such that $A$ is r.e., either $A \backslash M$ is $\mathscr{M}$-finite or $\mathscr{M} \backslash A$ is $\mathscr{M}$-finite;

(b) $\mathscr{M} \backslash M$ is not $\mathscr{M}$-finite.

The first construction of a maximal set (in $P$ ) was obtained in Friedberg [3]. Our first result implies that this construction cannot be carried out in $P^{-}+B \Sigma_{2}$. It is worthwhile to point out that because of the presence of nonstandard elements, the method of partitioning the universe recursively into $\omega$ disjoint pieces, such as that carried out in [7] for $\aleph_{\omega}^{L}$, is not applicable here, since $\mathscr{N}$ is not $\Sigma_{1}$ definable in $\mathscr{I}$. Thus although the presence of the function $f$ seems to indicate a strong resemblance in structure between $\mathscr{I}$ and $\boldsymbol{\aleph}_{\omega}^{L}$, there is a subtle difference between them making the argument in [7] not applicable in the present context. Instead an idea derived from Chong and Lerman [2] is appropriate here.

TheOREM 1. There is no maximal set in $\mathscr{I}$.

Proof. Let $M \subset \mathscr{I}$ be an r.e. set. Assume that $\mathscr{I} \backslash M$ is not $\mathscr{I}$-finite. We will produce an r.e. set $A$ containing $M$ such that neither $A \backslash M$ not $\mathscr{I} \backslash A$ is $\mathscr{I}$-finite.

It follows from Lemma 3 that $\bar{M}=\mathscr{I} \backslash M$ is not bounded in $\mathscr{I}$. Let $f: \mathscr{N} \rightarrow \mathscr{I}$ be a cofinal map recursive in $\emptyset^{\prime}$ (Lemma 2 (c)). Let $h: \mathscr{I} \times \mathscr{I}$ $\rightarrow \mathscr{I}$ be a recursive approximation of $f$, as given in Lemma 4 . It is not difficult to make $h$ non-decreasing in the following sense: $h(s, i) \leq h(t, j)$ whenever $s \leq t$ and $i \leq j$ (and both are defined). We assume in the following that $h$ is indeed non-decreasing. 
Let $M^{t}$ be the set of elements enumerated into $M$ by stage $t$. Let $m(0)$ be the least element of $\bar{M}$. For each $0<i$ in $\mathscr{N}$, first let $k^{*}$ be the least $k>i$ such that $m(i-1)<f(k)$. Let $m(i)$ be the least member of $\bar{M}$ greater than $f\left(k^{*}\right)$. Observe that since $\bar{M}$ is unbounded in $\mathscr{I}, M$ is regular, and $\mathscr{I}$ is a model of $B \Sigma_{2}$ (Lemma 2 (a)), we have by Proposition 1 (b) all the $m(i)$ 's to be well-defined. One may give a recursive approximation for the $m(i)$ 's at stage $s$ by setting $k^{*}$ to be the least $k$ such that $m(s, i-1)<h(s, k)$, and then choosing $m(s, i)$ to be the least $z$ in $\bar{M}^{s}$ such that $z>h\left(s, k^{*}\right)$. Then $m(s, j) \leq m(t, i)$ for $s \leq t$ and $j \leq i$.

For each standard $i$, we have $\lim _{s} m(s, i)=m(i)$. For nonstandard $i, \lim _{s} m(s, i)$ is not bounded in $\mathscr{I}$. Since $f(i)=a_{i}(i \in \mathscr{N})$, by Lemma $2(b)$ the correct value of $m(i)$ is reached by stage $m(i+1)$. This means that for all $s \geq m(i+1), m(s, i)=m(i)$. This property allows one to separate an $m(i)$ from those $m(j)$ 's which one wishes to avoid.

Define a function $S^{\prime}: \mathscr{I} \times \mathscr{I} \times \mathscr{I} \rightarrow 2$ as follows: Given $s$ and $j<i$, let $t(s)$ be the least $t$ such that $m(t, i)=m(s, i)$. If there is no $u<t(s)$ such that $m(u, i)=m(t(s), j)$, let $S^{\prime}(s, j, i)=0$. Otherwise, let $u(s)$ be the least such $u$ and set $S^{\prime}(s, j, i)=1-S^{\prime}(u(s), j, i)$. We claim that $S^{\prime}(s, j, i)$ $<2$ for all $s, j$ and $i$. Indeed suppose that $S^{\prime}(s, j, i) \geq 2$ for some $s, j$ and $i$. Then using $I \Sigma_{1}$, there exists a least $s$, for which there exist $j$ and $i$ such that $S^{\prime}(s, j, i) \geq 2$. Then $S^{\prime}(s-1, j, i)<2$ by assumption, so that the definition of $S^{\prime}$ specifies that $S^{\prime}(s, j, i)<2$, a contradiction. This proves the claim.

Observe that for each standard pair $j<i$, if $s_{1}>s$ and $m(s, i)=m\left(s_{1}, i\right)$ $=m(i)$ then $S^{\prime}(s, j, i)=S^{\prime}\left(s_{1}, j, i\right)$. On the other hand, if $j$ is standard and $i$ is nonstandard, let $v_{1}$ be the least $v$ such that $m(v, i)>m(v, j)=$ $m(j)$. Then for $t>v_{1}$ such that $m(t, i)>m\left(v_{1}, i\right)$, we have $m(t, j)=m(j)$, and so $S^{\prime}(t, j, i)=1-S^{\prime}\left(w_{1}, j, i\right)$, where $w_{1}$ is the least $w$ (if exists) such that $m(w, i)=m(j)$, or else $S^{\prime}(t, j, i)=0$. It follows that for each pair $(j, i)$ such that $j$ is standard and less than $i, \lim _{s} S^{\prime}(s, j, i)=S(j, i)$ exists (and is either 0 or 1 ). We need a lemma.

LEMma 5. Let $j$ be standard and suppose that $S^{\prime}(s, j, i)=S(j, i)$ where $j<i$. Then $m(s, i) \neq m(j)$.

Proof. First of all, assume that $i$ is standard. Suppose that $S^{\prime}(s, j, i)$ $=S(j, i)$ and that $m(s, i)=m(j)$. Let $t_{1}$ be the least $t>s$ such that $m(s, i)=m(i)$. At stage $t_{1}$, we have $m\left(t_{1}, j\right)=m(j)$. If we set $u_{1}$ to be 
the least $u$ such that $m(u, i)=m(j)$, then $u_{1} \leq s$ and $S^{\prime}\left(u_{1}, j, i\right)=S^{\prime}(s, j, i)$, so that by definition

$$
S^{\prime}\left(t_{1}, j, i\right)=1-S^{\prime}\left(u_{1}, j, i\right)=1-S^{\prime}(s, j, i)=1-S(j, i) .
$$

But $S^{\prime}\left(t_{1}, j, i\right)=S(j, i)$, and so we get a contradiction. Hence $m(s, i) \neq$ $m(j)$.

Now suppose that $i$ is nonstandard and that $m(s, i)=m(j)$. Then $\lim _{s} m(s, i)$ does not exist. Nevertheless, if $v_{1}$ is the least $v$ such that $m(v, i)>m(v, j)=m(j)$, then for $v>v_{1}$ such that $m\left(v_{1}, i\right)>m(v, i)$, we have $S^{\prime}(v, j, i)=S(j, i)=1-S^{\prime}\left(u_{1}, j, i\right)$, where $u_{1}$ is the least $u$ such that $m(u, i)=m(j)$, so that if $m(s, i)=m(j)$ then $S^{\prime}(s, j, i)=S^{\prime}\left(u_{1}, j, i\right) \neq S(j, i)$, a contradiction. Thus if we assume that $S^{\prime}(s, j, i)=S(j, i)$, then $m(s, i)$ $\neq m(j)$.

Choose $D \subset \mathscr{N}$ such that $D$ is the set of triples of the form $(j, i, S(j, i))$, where $j$ is odd, $i$ is even and $j<i$. By Lemma 1 there exists an $\mathscr{M}_{0}$-finite set $K$ whose standard part is $D$. As observed in the proof of Lemma 4, $\mathscr{I}$ is a $\Sigma_{1}$ elementary substructure of $\mathscr{M}_{0}$, and so is closed under the coding function. In particular, $K$ is $\mathscr{I}$-finite. Before we show that $M$ is not maximal, let us prove a lemma.

Lemma 6. There is an $\mathscr{I}$-finite subset $K_{1}$ of $K$ containing $D$ such that: For each even $i$, if $\left(j_{1}, i, r_{i}\right) \in K_{1}$ for some odd $j_{1}$ and some $r_{1}$, then for all standard odd $j<i$, there is an $r$ such that $(j, i, r)$ is in $K_{1}$. Furthermore, for each such $j$,

(a) If $i$ is standard, then $r=S(j, i)$;

(b) $r$ is the unique $n$ such that $(j, i, n)$ belongs to $K_{1}$;

(c) $m(s, i) \neq m(j)$ whenever $S^{\prime}(s, j, i)=r$.

Proof. Let $K_{0}$ be the set of $(j, i, n)$ in $K$ such that $n$ is the least $r<2$ (if exists) satisfying $(i, j, r) \in K$ for a given $(i, j)$. Then $K_{0}$ is $\mathscr{I}$-finite. We show that all the statements, except possibly (c), already hold in $K_{0}$.

If $i$ is standard, then $(j, i, S(j, i))$ belongs to $D$ for each odd $j<i$, and is therefore in $K_{0}$ (since the standard part of $K$ is $D$ ). The definition of $D$ makes it clear that $S(j, i)$ is the only $n$ giving $(j, i, n)$ in $K_{0}$. Applying Lemma 5 gives (a)-(c) for standard $i$. Thus suppose that $i$ is nonstandard. To prove (b), let us briefly review the construction of the model $\mathscr{M}_{0}$ of Lemma 1 introduced in [9]. The discussion in the next paragraph is an expansion of the observation that $\mathscr{M}_{0}$ is sufficiently saturated. 
Let $V=V_{\omega+\omega}$, the collection of sets of rank less than $\omega+\omega$. Let $\mathscr{U}$ be a nonprincipal ultrafilter on $\omega$. Let $V^{*}$ be the ultrapower of $V$ modulo $\mathscr{U}$. There is an embedding $g: a \mapsto c_{a}$ of $V$ into $V^{*}$, where $c_{a}$ is the equivalence class of functions on $\omega$ containing the function with constant value $a$. The structure $\mathscr{M}_{0}$ is taken to be $g(\mathscr{N})$. It is not difficult to show that $g$ is an isomorphism on $\mathscr{N}$ so that $\mathscr{N}$ may be considered to be a bounded subset of $g(\mathscr{N})$. Let id be the equivalence class of functions containing the identity map on $\mathscr{N}$. Then $\mathscr{M}_{0} \vDash \mathrm{id}>c_{n}$ for all $n \in \mathscr{N}$, so that id is a nonstandard element of $\mathscr{M}_{0}$. Now given $E \subset \mathscr{N}, K^{*}=g(E) \cap$ id is coded in $\mathscr{M}_{0}$ so that it is $\mathscr{M}_{0}$-finite (hence $\mathscr{I}$ finite since $\mathscr{I}$ is closed under the coding function). Furthermore, the standard part of $K^{*}$ is $E$. Thus we see that a triple $(j, i, r)$ belongs to $K^{*}$ if and only if there is a member $X$ of $\mathscr{U}$ such that for all $k \in X$, $(j(k), i(k), r(k))<k$ and $(j(k), i(k), r(k)) \in E$, where $j(k), i(k)$ and $r(k)$ are the values of the $k$ th co-ordinates of $j, i$ and $r$ respectively, and where we assume that all finite tuples are coded by a natural number. In particular, this implies that if $(j, i, r) \in K^{*}$ then $j(k), i(k), r(k) \in E$ for almost all $k$ ('almost' in the sense of the ultrafilter $\mathscr{U}$ ). Let $E$ be the set $D$, then $K^{*}$ is just the set $K$ defined earlier.

Recall firstly that the set $D_{1}=\{S(j, i) \mid i, j$ are standard $\}$, where $j$ is odd and $i$ is even, is a subset of 2 . Now suppose that $\left(j_{1}, i, r_{1}\right) \in K_{0}$ for some odd $j_{1}$ and some $r_{1}$. Let $j$ be a fixed standard odd number. Then we have $\left(j, i(k), S(j, i(k)) \in D\right.$ for almost all $k$. By the finiteness of $D_{1}$ there is therefore an $X \in \mathscr{U}$ and a fixed $r<2$ such that $X=\{k \mid(j, i(k), r) \in D\}$, where $r=S(j, i(k))$ for all $k$ in $X$.

Suppose that $(j, i, n) \in K_{0}$ for some standard $n$. Then $(j, i(k), n(k))$ is in $D$ almost everywhere. This means that $n(k)=S(j, i(k))$ almost everywhere, and so $r=n$. This shows that for each standard odd $j$ there is a unique $r$ such that $(j, i, r) \in K_{0}$. Thus (b) follows as a consequence.

We will define a $K_{1} \subset K_{0}$ and prove that (c) holds. Consider now $i$ as ranging over the even numbers. Given a standard odd number $j$, let $(j, i, r)$ be in $K_{0}$ and define

$$
\Gamma_{j}=\left\{i \mid i \text { is even and }(\exists s)\left[S^{\prime}(s, j, i)=r \text { and } m(s, i)=m(j)\right\} .\right.
$$

Then $\Gamma_{j}$ is $\Sigma_{1}$ over $\mathscr{I}$ and if it is nonempty, it has a least element $\gamma_{j}$. Furthermore, by (a) and Lemma 5 the least element $\gamma_{j}$ is nonstandard. Without loss of generality, let us assume that $\gamma_{j}$ is defined for each 
standard odd $j$.

Claim. There is a nonstandard number $b$ such that $b<\gamma_{j}$ for all $\gamma_{j}$.

Let $\gamma_{j}$ be in the equivalence class (modulo $\mathscr{U}$ ) of the function $f_{j}: \mathscr{N} \rightarrow$ $\mathscr{N}$. For each $k \in \mathscr{N}$, let $Y_{k} \in \mathscr{U}$ such that for all $n \in Y_{k}$, we have $f_{j}(n)>k$ for odd $j \leq k$. Basic properties of ultrafilters ensure that $Y_{k}$ exists. It is also possible to arrange $Y_{k} \supset Y_{k+1}$. We assume that this is indeed done. Set $b_{k}(n)=\min _{j \leq k}\left\{f_{0}(n)\right\}$ for $n \in Y_{k}$.

Let $Y=\cap_{k} Y_{k}$, Suppose that $Y \neq \emptyset$. Then for all $n$ in $Y$, and for all standard $k$, we have $f_{j}(n)>k$ whenever $j<k$. This implies that $f_{j}(n)$ is not a finite number, which is not possible. Thus $Y=\emptyset$. It follows that for every $n$ there is a largest $k$ such that $n \in Y_{k}$. Set

$$
b(n)= \begin{cases}b_{k_{1}}(n), & k_{1} \text { is the greatest } k \text { with } n \in Y_{k} . \\ 0 & \text { otherwise. }\end{cases}
$$

For each $k$, we then have $b(n)=b_{k_{1}}(n)>k$ whenever $n \in Y_{k}$. Furthermore for each $j, b(n) \leq f_{j}(n)$ for $n$ in $Y_{k}$, where $k^{*}>j$. Hence in $\mathscr{I}$ the element $b$ is nonstandard and less than each $\gamma_{j}$. This proves the claim.

Now let $b$ be as specified in the Claim. Let $K_{1} \subset K_{0}$ such that $(j, i, r) \in K_{1}$ if and only if $(j, i, r) \in K_{0}$ and $i<b$. Then by the choice of $b$, we have $(j, i, r) \in K_{1}$ for every standard even number $i$, and that $r=S(j, i)$. Furthermore, if $i$ is nonstandard with $j$ standard and $(j, i, r) \in K_{1}$, then $S^{\prime}(s, j, i)=r$ implies that $m(s, i) \neq m(j)$. Thus $(c)$ is true.

We are now ready to show that $M$ is not maximal. Let

$$
\left.A=M \cup\left\{m(s, i) \mid(\forall j)(\forall r)[(j, i, r)) \in K_{1} \rightarrow S^{\prime}(s, j, i)=r\right]\right\} .
$$

Clearly $A$ contains $M$ as a subset. By Lemma 6(a) and (b), if $i$ is a standard even number then $(j, i, S(j, i))$ is in $K_{1}$ and $S(j, i)$ is the unique $r$ giving $(j, i, r)$ in $K_{1}$. Since $m(s, i)=m(i)$ and $S^{\prime}(s, j, i)=S(j, i)$ for all sufficiently large $s$, we have $m(i) \in A$ for each standard even $i$. Thus $A \backslash M$ is not $\mathscr{I}$-finite. Since $m(s, i)$ is enumerated in $A$ if and only if $S^{\prime}(s, j, i)=r$ for all $(j, i, r) \in K_{1}$, we see by Lemma 5 and Lemma 6 that setting $j$ odd and standard ensures that $m(s, i) \neq m(j)$. Thus $A$ avoids each $m(j)$ where $j$ is standard and odd. This shows that $M$ is not maximal and proves Theorem 1.

Corollary 1. $P^{-}+B \Sigma_{2}$ does not prove the existence of a maximal set. 
We thank T. Slaman for pointing out to us the next corollary:

CoRollary 2. There is no finite injury type construction of a maximal set.

Proof. By Slaman and Woodin [10], all finite injury priority arguments can be carried out in models of $I \Sigma_{1}$. Hence if maximal sets can be constructed using a finite injury argument, it would exist in every model of $P^{-}+I \Sigma_{1}$, and in particular, in the model $\mathscr{I}$ of Theorem 1 . But this is not possible.

\section{Existence of Maximal Sets}

In this section we construct a maximal set for every model of $\mathrm{P}^{-}+$ $I \Sigma_{3}$, and show that there is a model of $P^{-}+\neg B \Sigma_{3}+I \Sigma_{2}$ with a maximal set. The method of $e$-state introduced in Friedberg [3], appropriately modified, will be used.

TheOREm 2. Let $\mathscr{M}$ be a model of $P^{-}+I \Sigma_{3}$. Then there is a maximal set $M$ in $\mathscr{M}$.

Proof. Given $e \in \mathscr{M}$, we say that $x$ is in the $j$ th $e$-state at stage $s$ if

$$
j=\Sigma\left\{2^{e-i} \mid x \in W \text { and } i \leq e\right\} .
$$

Observe that for each $e \in \mathscr{M}$, there are only $\mathscr{M}$-finitely many $e$-states. Let $c(s, i)$ be the ith element of $\bar{M}^{s}$, the complement of $M$ in $\mathscr{M}$ at stage $s$ (i.e. $c(s, i)$ is the element $y$ of $\bar{M}^{s}$ such that $y \cap \bar{M}^{s}$ is in one-one correspondence with $i$ ).

We begin by setting $M^{0}=\emptyset$. At stage $s+1$, let $j(s+1,0)$ be the largest $j$ which is a 0 -state at stage $s+1$, and for which there exists an $x \geq c(s, 0)$ in $\bar{M}^{s}$ which belongs to the $j$ th 0 -stage. The largest $j$ as required by $j(s+1,0)$ exists. To see this, observe that since there are only $\mathscr{M}$-finitely many 0 -states, there are therefore only $\mathscr{M}$-finitely many $j$ 's for which there exist $x \geq c(s, 0)$ in $\bar{M}^{s}$ and in the jth 0 -state. Hence there is a least $j_{1}$ (by Proposition 1 (b)) which is greater than all such 0 -states. Then $j_{1}-1$ is the largest 0 -state at stage $s+1$ as described above. Let $c(s+1,0)$ be the least $x \geq c(s, 0)$ with 0 -state $j(s+1,0)$.

By induction, suppose that $c(s+1, e-1)$ is defined. Let $j(s+1, e)$ be the largest $j$ which is an $e$-state at stage $s+1$, and for which there exists an $x \leq s+1$ in $\bar{M}^{s}$ with

(5) $x>\max \{c(s+1, d) \mid d, e\}$; 
(6) $x \geq c(s, e)$;

(7) $x$ is in the $j$ th $e$-state.

Again Proposition 1 (b) justifies the existence of $j(s+1, e)$. Let $c(s+1, e)$ be the least $x$ satisfying (5)-(7), with $j$ in (7) set to be $j(s+1, e)$. Let

$$
M^{s+1}=M^{s} \cup\{x \mid(\exists e)[(c(s+1, e)<x<c(s+1, e+1)]\} .
$$

Let $M=\cup_{s} M^{s}$. We show that $M$ is maximal.

LEMMA 7. For each e, $\lim _{s} c(s, e)=c_{e}$ exists.

Proof. We prove this by induction. Let $e$ be given. Suppose that $c_{d}$ exists for all $d<e$. The map $d \mapsto c_{d}$ is $\Sigma_{2}$ over $\mathscr{M}$. Since $B \Sigma_{2}$ holds in $\mathscr{M}$ (Proposition 1 (c)), there is a stage $s_{0}$ such that for all $s \geq s_{0}$, $c(s, d)=c_{d}$ for all $d<e$. After stage $s_{0}$, there is a largest $e$-state $j$ such that at some stage $s>s_{0}, j=j(s+1, e)$. This follows from the fact that there are only $\mathscr{M}$-finitely many $e$-states, so that the set

$$
\{j \mid(\exists s)[j=j(s+1, e)]\}
$$

is $\mathscr{M}$-finite and has a largest value which we denote as $j^{*}$. Let the corresponding stage $s$ be denoted $s^{*}$. Then at stage $s^{* \prime}$, the construction sets $c\left(s^{* \prime}, e\right)$ to be the least $x$ satisfying (5)-(7) with $j$ in (7) chosen to be $j\left(s^{* \prime}, e\right)=j^{*}$. Then for all stages $s$ greater than $s^{*}$, the $j^{*}$ th $e$-state is the highest $e$-state and so $c(s, e)=c\left(s^{* \prime}, e\right)$. Hence $\lim _{s} c(s, e)=c_{e}$ exists.

By $I \Sigma_{2}$, we conclude that $c_{e}$ exists for each $e \in \mathscr{M}$.

Let $e$ be fixed. Define a $\Sigma_{3}$ relation as follows:

$$
\begin{array}{r}
\theta(e) \longleftrightarrow(\exists d)\left\{\left[(\forall b \geq d)(\forall s)(\exists t \geq s)(c(t, b)) \in W_{e}^{t}\right)\right] \vee \\
\left.\left[(\forall b \geq d)(\forall s)(\exists t \geq s)\left(c(t, b) \notin W_{e}^{t}\right)\right]\right\} .
\end{array}
$$

In view of Lemma $7,(8)$ is equivalent to

$$
\theta(e) \longleftrightarrow(\exists d)\left[(\forall b \geq d)\left(c_{b} \in W_{e}\right) \vee(\forall b \geq d)\left(c_{b} \notin W_{e}\right)\right] .
$$

LEMma 8. $\theta(e)$ holds for all $e$.

Proof. Suppose that $\theta(e)$ is false for some $e$. By $I \Sigma_{3}$, there is a least $e$ for which $\neg \theta(e)$ holds. Denote this by $e_{0}$. Since $\theta(e)$ holds for all $e<e_{0}$, we see that for each such $e$, there is a least $d$ witnessing the truth of $\theta(e)$. By $I \Pi_{2}$ let $d(e)$ be the least such $d$. The map $\epsilon \mapsto d(e)$ is then $\Sigma_{3}$. 
In fact $d(e)$ is the $d$ which satisfies $[\cdots]$ in (8) and such that $d-1$ fails to satisfy [...]. Now apply $B \Sigma_{3}$ to establish an upper bound $a_{0}$ for the $d(e) ' s, e<e_{0}$.

Thus for $b_{1}$ and $b_{2}>a_{0}$, and $e<e_{0}$, we have $c_{b_{1}} \in W_{e}$ if and only if $c_{b_{2}} \in W_{e}$. This implies that $c_{b_{1}}$ and $c_{b_{2}}$ lie in the same $j$ th $e$-state for some $j$. Now choose $b_{1}<b_{2}$, both greater than $a_{0}$, such that $c_{b_{1}} \notin W_{e_{0}}$ and $c_{b_{2}} \notin W_{e_{0}}$. This implies that $c_{b_{2}}$ has higher $e_{0}$-state than $c_{b_{1}}$ at all stages $s$ where $c\left(s, b_{1}\right)=c_{b_{1}}, c\left(s, b_{2}\right)=c_{b_{2}}$ and $c_{b_{2}} \in W_{e_{0}}^{s}$. The process of maximizing $e_{0}$-states then requires that for such $s, j\left(s, e_{0}\right) \geq j^{*}$, where $j^{*}$ is the $e_{0}$-state of $c_{b_{2}}=c\left(s, b_{2}\right)$. But this implies that for such $s, c(s, b) \geq c_{b_{2}}$, contradicting our assumption on $s$.

LEMMA 9. $M$ is maximal.

Proof. First of all $\mathscr{M} \backslash M$ is not $\mathscr{M}$-finite since this is the set $\left\{c_{e} \mid e \in \mathscr{M}\right\}$. Suppose that $W_{e} \backslash M$ is not $\mathscr{M}$-finite. By Lemma 3 this means that $W_{e} \backslash M$ is not bounded. By Lemma $8, \theta(e)$ holds and so (8) implies that for all sufficiently large $b$, we have $c_{b} \in W_{e}$. Thus $\mathscr{M} \backslash\left(M \cup W_{e}\right)$ is bounded below and hence $\mathscr{M}$-finite by Lemma 3 .

CoRollary 3. The existence of maximal sets can be proved in the system $P^{-}+I \Sigma_{3}$.

We do not know if $I \Sigma_{3}$ can be strengthened to $I \Sigma_{2}$ in the above corollary. It is not difficult to note that the maximal set construction given in the proof of Theorem 2 can be carried out in $I \Sigma_{2}$, and that Lemma 7 is true (Shore and Slaman had observed that, with a little bit more work, $I \Sigma_{1}$ is sufficient to achieve these), although the maximality of $M$ does not follow as a consequence. Nevertheless, we have the following partial result which indicates that the existence of a maximal set is consistent with the assumption of $I \Sigma_{2}$, even in the presence of $\neg B \Sigma_{3}$ (hence $\neg I \Sigma_{3}$ ).

Theorem 3. There is a model of $P^{-}+\neg B \Sigma_{3}+I \Sigma_{2}$ with a maximal set.

Proof. Let $\mathscr{M}$ be a model of full Peano arithmetic. By Kirby and Paris [6] there is a $\Sigma_{3}$ elementary substruture $\mathscr{M}_{0}$ of $\mathscr{M}$ which satisfies $P^{-}+\neg B \Sigma_{3}+I \Sigma_{2}$. This model is obtained by choosing a nonstandard element $a$ in $\mathscr{M}$, and then closing off in $\mathscr{M}$ under $\Sigma_{3}$ Skolem functions. Now 
perform the construction of a maximal set $M$ in $\mathscr{M}$, as described in the proof of Theorem 2. The same $\Sigma_{1}$ algorithm (which is parameter free) constructs an r.e. set $M_{0}$ in $\mathscr{M}_{0}$ as well. Let $c(s, b)$ denote the $b$ th 'marker' position at stage $s$ (as defined in the proof of Theorem 2) in the construction of $M$. For $b$ and $s$ in $\mathscr{M}_{0}$, the function $c(s, b)$ is $\Sigma_{1}\left(\mathscr{M}_{0}\right)$ and so gives the $b$ th marker position at stage $s$ in the construction of $M_{0}$. Then as mentioned earlier, Lemma 7 holds in $\mathscr{M}_{0}$ for $M_{0}$. The maximality of $M$ in $\mathscr{M}$ implies that the $\Sigma_{3}$ sentence $\theta(e)$ of (8) is true in $\mathscr{M}$ for each $e$ in $\mathscr{M}_{0}$. Being a $\Sigma_{3}$ elementary substructure, this sentence is true in $\mathscr{M}_{0}$ as well. But then by Lemma 7 this means that for each $e$ in $\mathscr{M}_{0}$, there is $d$ such that either $c_{b} \in W_{e}$ for all $b \geq d$, or $c_{b} \notin W_{e}$ for all $b \geq d$, Since $M_{0}=\mathscr{M}_{0} \backslash\left\{c_{b} \mid b \in \mathscr{M}_{0}\right\}$, we see that $M_{0}$ is maximal.

\section{Maximal sets and $\Sigma_{1}$ indcution}

Lemma 10. There is a model $\mathscr{J}$ of $P^{-}+I \Sigma_{0}+\neg B \Sigma_{1}$ with a $\Sigma_{2}$ injection $p$ from $\mathscr{J}$ into an infinite subset of $\mathscr{N}$.

Proof. Let $\mathscr{M}$ be a nonstandard model of full Peano arithmetic. Let $b_{0}$ be a nonstandard element of $\mathscr{M}$. Let $(\exists x) \varphi(e, x, z, y)$ be a universal $\Sigma_{1}$ predicate, where $\varphi$ is $\Sigma_{0}$. Let $\mathscr{J}_{0}=\left\{0, b_{0}\right\}$. Let $\mathscr{J}_{n+1}$ be the hull of $\mathscr{J}_{n}$ in $\mathscr{M}$ with respect to the first $n+1 \Sigma_{1}$ functions. This means that

$$
\mathscr{J}_{n+1}=\left\{a \mid \mathscr{M} \vDash(\exists x)\left(\exists z \in \mathscr{J}_{n}\right)(\exists e \leq n+1) \varphi(e, x, z, a)\right\} .
$$

We call $z$ a parameter of $a$ in $\mathscr{J}_{n}$. Let $\mathscr{J}=\cup \mathscr{J}_{n}$. Then $\mathscr{J}$ is countable and is a $\Sigma_{1}$ elementary substructure of $\mathscr{M}$ (although it is not an initial segment of $\mathscr{M}$ ). Furthermore, $\mathscr{N} \subset \mathscr{J}$.

Let $X$ be a nonempty $\Sigma_{0}$ set defined over $\mathscr{J}$ by the $\Sigma_{0}$ formula $\chi(x)$. Then in $\mathscr{M}$ there is a $\Sigma_{0}$ set defined by the same formula with a least element $a$. Then

$$
\mathscr{M} \vDash(\exists z)[\chi(z) \text { and }(\forall y<z) \neg \chi(y)]
$$

holds. Being a $\Sigma_{1}$ elementary substructure, $\mathscr{J}$ also satisfies the same statement, so that there is a least element with property $\chi$. Hence $\mathscr{J}$ is a model of $P^{-}+I \Sigma_{0}$.

We show that $\mathscr{J} \vDash \neg B \Sigma_{1}$ (cf. [6]). Let $\varphi$ be as above. Then for each $a \in \mathscr{J}$, the sentence $\psi(a)$ saying

$$
\begin{aligned}
\left(\exists e<b_{0}\right)(\exists u)[\varphi(e, u(0), u(1), u(2)) \text { and } u(2) \\
\quad=a \text { and }(\forall z<u) \neg \varphi(e, z(0), z(1), z(2))]
\end{aligned}
$$


is $\Sigma_{1}$ in $\mathscr{M}$, where $u \mapsto\left((u(0), u(1), u(2))\right.$ is a $\Sigma_{0}$ coding function (for triples). Since $\psi(a)$ is true in $\mathscr{M}$, it is true in $\mathscr{J}$ as well. Thus for $a<b_{0}+1$, there exist $e<b_{0}$ and $u$ to satisfy $\psi(a)$. If $\mathscr{J} \vDash B \Sigma_{1}$, there would exist a uniform upper bound $u^{*}$ on the set of such $u^{\prime}$ s. This in trun implies that $\left(\forall a<b_{0}+1\right) \psi(a)$ is $\Sigma_{1}(J)$, and hence $\Sigma_{1}(\mathscr{M})$ and true in $\mathscr{M}$. A pigeon hole argument in $\mathscr{M}$ then says that there exist $u_{1}$ and $u_{2}$ and an $e$ such that $\psi\left(u_{1}(2)\right)$ and $\psi\left(u_{2}(2)\right)$ hold for the same $e$. This is not possible since $u_{1} \neq u_{2}$ and yet both are least for the same $e$.

Define an approximation to the $\mathscr{J}_{n}$ 's as follows: $\mathscr{J}_{0, s}=\left\{0, b_{0}\right\}$ for all s. For $d>0, a \in \mathscr{J}_{d, s}$ if and only if there is an $e \leq d$ and $a z_{0} \in \mathscr{J}_{d-1, s-1}$ such that $(\exists x<s) \varphi\left(e, x, z_{0}, a\right)$ holds. Then $(d, s) \mapsto J_{d, s}$ is uniformly $\Sigma_{1}$ in $\mathscr{J}$. Furthermore, $\lim _{s} \mathscr{J}_{n, s}=\mathscr{J}_{n}$ is finite for each $n \in \mathscr{N}$. We now define a $\Sigma_{2}$ function from $\mathscr{J}$ into $\mathscr{N}$. Let $p^{\prime}(s, 0)=0$ and $p^{\prime}\left(s, b_{0}\right)=1$ for all $s$, and initially set $p^{\prime}(0, a)=0$ for all $a$ in $\mathscr{J}$. Let $s>0$ and $d>0$. Suppose that every element in $\mathscr{J}_{a, s}$ is defined under $p^{\prime}$. Let $a \in \mathscr{J}_{a+1, s}$. Choose the least $e \leq d+1$ such that

$$
(\exists u<s)[\varphi(e, u(0), u(1), u(2)) \text { and } u(2)=a]
$$

holds, with $u(1) \in \mathscr{J}_{d, s}$ (hence $p^{\prime}(s, u(1))$ is defined). Let $e^{*}$ be this least $e$, and let $p^{\prime}(s, a)=\left(e^{*}, p^{\prime}\left(s, u^{*}(1)\right)\right)$, where $u^{*}$ is selected to be the least $u<s$ such that $u(1)$ is the least element in satisfying (10). Then $p^{\prime}$ is a partial function. Now $p(0)=\lim _{s} p(s, 0)=0$ and $p\left(b_{0}\right)=\lim _{s} p^{\prime}\left(s, b_{0}\right)=1$ by prescription. Suppose that every member of $\mathscr{J}_{n}$ is defined under $p$ and belongs to $\mathscr{N}$. Let $a \ni \mathscr{J}_{n+1} \backslash \mathscr{J}_{n}$ be defined by the least index $e_{a}$ with a least parameter $z_{a}$ in $\mathscr{J}_{n}$. Then $p^{\prime}(s, a)$ is defined, and is equal to $\left(e_{a}, p\left(z_{a}\right)\right.$ ), for all sufficiently large $s$. Hence for each $a \in \mathscr{J}$, we have $\lim _{s} p^{\prime}(s, a)=p(a)$ to be defined and belongs to $\mathscr{N}$. Suppose that distinct members of $\mathscr{J}_{n}$ are mapped to distinct members of $\mathscr{N}$ by $p$. Let $a_{1} \neq a_{2}$ be in $\mathscr{J}_{n+1}$. Then either the least $e$ (denoted $e_{a_{1}}$ and $e_{a_{2}}$ respectively) used to define them (in $\varphi(e, x, z, y)$ ) are different or else $e_{a_{1}}=e_{a_{2}}$ but different least parameters $z_{a_{1}}$ and $z_{a_{2}}$ are used respectively in the definition. In either case we clearly have $p\left(a_{1}\right)=\left(e_{a_{1}}, p\left(z_{a_{1}}\right)\right) \neq\left(e_{a_{2}}, p\left(z_{a_{2}}\right)\right)=p\left(a_{2}\right)$.

Now suppose that there are two distinct elements of $\mathscr{J}$ which have the same image under $p$. By the above argument, these two elements come from $\mathscr{J}_{i}$ and $\mathscr{J}_{n}$, with $i<n$. Take the least $i$, denoted $i_{0}$ with such an $n$, and then choose the least $n$, denoted $n_{0}$, for that $i_{0}$. Suppose that $a \in \mathscr{J}_{i_{0}}$ ann $b \in \mathscr{J}_{n_{0}}$ are mapped to the same image under $p$. Then they 
must be defined by $\varphi$ via the same index $e$, but with different least parameters. These parameters (say $z_{a}$ and $z_{b}$ ), however, reside in $\mathscr{J}_{i_{0}-1}$ and $\mathscr{J}_{n_{0}-1}$ respectively, and by the choice of $i_{0}$ and $n_{0}$, have different images under $p$. Since $p(a)=\left(r, p\left(z_{a}\right)\right)$ and $p(b)=\left(e, p\left(z_{b}\right)\right)$, we see that $p(a) \neq p(b)$, a contradiction. Hence $p$ is an injection.

Theorem 4. There is a model of $P^{-}+\neg I \Sigma_{1}$ with a maximal set $M$.

Proof. Let $\mathscr{J}$ be the model constructed in Lemma 10. We 'invert' the $\Sigma_{0}$ function $p^{\prime}$ as follows:

$$
g^{\prime}(s, e)= \begin{cases}a & \text { if } a<s \text { and } p^{\prime}(s, a)=e, \\ \text { undefined } & \text { otherwise }\end{cases}
$$

Then $g^{\prime}$ is $\Sigma_{0}$ and for all $e$ in the range of $p, g(e)=\lim _{s} g^{\prime}(s, e)=p^{-1}(e)$. We construct a maximal set $M$ using an $e$-state method. However, since we are working in a model of $\Sigma_{0}$, the exponential function is not available at our disposal, and so the $e$-states will be defined in a somewhat roundabout way.

The requirements will be indexed by $\mathscr{N}$ via the $\operatorname{map} \lim _{s} g^{\prime}(s, \cdot)=g$. These requirements are:

$$
R_{a}: \mathscr{J} \backslash\left(M \cup W_{a}\right) \text { is } \mathscr{J} \text {-finite or } W_{a} \backslash \bar{M} \text { is } \mathscr{J} \text {-finite. }
$$

Requirement $R_{a}$ is indexed by the number $p(a)$ in $\mathscr{N}$, and $W_{a}$ will have higher priority than $W_{b}$ if $p(a)<p(b)$. As for many finite or infinite injury argument in $\alpha$ recursion theory where the true priority of a requirement is known only in the limit, we also have here that for all sufficiently large $s$, the priority of $R_{g^{\prime}(s, e)}$ at stage $s$ is $e$, whenever $e$ is in the image of $p$.

For each $e$, the $e$-state of an element $x$ at stage $s$ is defined to be the $\Sigma_{0}$ set

$$
X(s, e, x)=\left\{i \mid i \leq e \text { and } x \in W_{g^{\prime}(s, i)}^{s}\right\} .
$$

whenever $g^{\prime}(s, i)$ is defined. We also say that $x$ is in the $e$-state $X(s, e, x)$. A $\Sigma_{0}$ set $K$ is said to have a higher $e$-state than another $\Sigma_{0}$ set if the least number which is in one but not the other set belongs to $K$. Notice that for each $s$ and $e$, it is a $\Sigma_{0}(\mathscr{J})$ procedure to decide if there exists a highest $e$-state within $s$ step of computation. Set $M^{0}=\emptyset$ and $c(0, e)=0$ for all $e$. Suppose that $c(s, i)$, for $i \leq s$, is defined and belongs to $\bar{M}^{s}$, 
with the property that for all $x$ satisfying $c(s, i)<x<c(s, i+1), x \in M^{s}$. Compute $s+1$ steps to search for the set $K$ of highest 0 -state (if exists) such that (*): $K$ is a 0 -state for an $x \geq \max \left\{g^{\prime}(s+1,0), s(s, 0)\right\}$ in $\bar{M}^{s}$, and less than or equal to $s+1$. If such a $K$ does not exist, let $c(s+1,0)$ $=c(s, 0)$. Otherwise, let $K(s+1,0)$ be such a set. Then the collection of $x$ 's satisfying (*) with $K$ there replaced by $K(\mathrm{~s}+1,0)$ is $\Sigma_{0}$, and so has a least element. This is set to be $c(s+1,0)$.

Now assume that $c(s+1, e)$ is defined. Perform $s+1$ steps of computation to choose $K(s+1, e+1$ ) (if exists) to be the highest $e+1$ state for an $x \leq s+1$ satisfying (5), and (6*), where (6*) says: $x \geq$ $\max \left\{g^{\prime}(s+1, e+1), c(s, e+1)\right\}$. If $K(s+1, e+1)$ does not exist, let $c(s+1, e+1)$ be the least element in $\bar{M}^{s+1}$ greater than $c(s+1, e)$. Otherwise, use $L \Sigma_{0}$ to choose the least $x$ satisfying (5) and (6*), and such that $K(s+1, e+1)$ is an $e+1$-state for $x$. Set

$$
M^{s+1}=M^{s} \cup\{x \mid(\exists e<s+1)[c(s+1, e)<x<c(s+1, e+1)]\} .
$$

Define $M=\cup_{s} M^{s}$.

Lemma 11. For each $e \in \mathscr{N}, \lim _{s} c(s, e)=c_{e}$ exists.

Proof. Let $e_{0}$ be in $\mathscr{N}$, and suppose that for $e<e_{0}, \lim _{s} c(s, e)=c_{e}$ exists. Since $\left\{e \mid e<e_{0}\right\}$ is finite, there is a stage $s_{0}$ such that for all $s \geq s_{0}, c(s, e)=c_{e}$ for each $e<e_{0}$. Choose $s_{1} \geq s_{0}$ such that for all $e \leq e_{0}$ in the image of $p$ (hence in the domain of $g$ ), $g^{\prime}(s, e)=g^{\prime}\left(s_{1}, e\right)$ whenever $s \geq s_{1}$. Then for all $s \geq s_{1}, W_{g^{\prime}(s, e)}=W_{p-1(e)}$ if $e$ is in the range of $p$, so that at most $W_{0}, W_{p-1(0)}, \cdots, W_{p-1\left(e_{0}\right)}$ are the only r.e. sets considered (if $p^{-1}(e)$ is defined for each $\left.e, 0 \leq e \leq e_{0}\right)$ for the maximizing of $e_{0}$-states after stage $s_{1}$. Thus in any case only finitely many r.e. sets are involved in this process, and for $s \geq s_{1}$, the maximal $e_{0}$-state $K\left(s, e_{0}\right)$ always exists. It follows that the position of $c\left(\cdot, e_{0}\right)$ may change at most finitely many times after stage $s_{1}$ to maximize $e$-states. Thus $\lim _{s} c\left(s, e_{0}\right)=c_{e_{0}}$ exists.

Induction on $\mathscr{N}$ shows that $c_{c}$ exists for each natural number $e$ in the image of $p$.

The construction shows that $\bar{M}$ has order type $\omega$ and is uhbounded in $\mathscr{J}$ (since $c_{e} \geq g(e)$ for each $e$ in the range of $p$ ). Thus if $W_{p^{-1(e)}} \backslash M$ is not $\mathscr{J}$-finite, it is not bounded in $\mathscr{J}$. The next result completes the proof of Theorem 4.

LEMMA 12. $M$ is maximal. 
Proof. By induction on $\mathscr{N}$. Let $e_{0}$ be in the image of $p$. Suppose that for all $e<e_{0}, R_{p^{-1}(e)}$ is satisfied whenever $p^{-1}(e)$ is defined. Choose an $x_{0}$ such that for all $e<e_{0}$, either $W_{p-1(e)} \backslash M \subset x_{0}$ or $\mathscr{J} \backslash\left(W_{p-1(e)} \cup M\right) \subset x_{0}$ (if $p^{-1}(e)$ is defined). Let $W_{p^{-1\left(e_{0}\right)}} \backslash M$ be non- $\mathscr{J}$-finite. If $\mathscr{J} \backslash\left(W_{p^{-1\left(e_{0}\right)}} \cup M\right)$ is not $\mathscr{J}$-finite (hence not bounded), there would exist natural numbers $v>r>e_{0}$ in the range of $p$ such that $c_{v} \in W_{p^{-1\left(e_{0}\right)}}, c_{r} \notin W_{p^{-1\left(e_{0}\right)}}$, and $c_{v}>$ $c_{r}>x_{0}$. Choose $s_{1}$ such that for all $s \geq s_{1}$, we have $g(e)=g^{\prime}(s, e)=p^{-1}(e)$ and $c(s, e)=c_{e}$ for each $e \leq v$ in the range of $p$. Then for all $s>s_{1}, c_{v}$ is in a higher $e_{0}$-state than $c_{r}$ is, and the principle of maximizing $e_{0}$-states demands that $c_{r}$ be discarded in preference for a number such as $c_{v}$ with higher $e_{0}$-state. This however contradicts the choice of $c_{r}$. Hence $\mathscr{J} \backslash\left(W_{p-1\left(e_{0}\right)} \cup M\right)$ is $\mathscr{J}$-finite. This proves the lemma.

Corollary 4. There is a model of $P^{-}+\neg B \Sigma_{2}$ with a maximal set.

Proof. By Proposition 1 (c) and Theorem 4.

CoRollary 5. The existence of a maximal set is strictly weaker than $I \Sigma_{3}$ and does not imply, nor is it implies by, either $I \Sigma_{1}$ or $B \Sigma_{2}$.

Proof. Corollary 3 and Theorem 3 show that the sentence on the existence of maximal sets is strictly weaker than $I \Sigma_{3}$. Corollary 1 and Corollary 4 show that $B \Sigma_{2}$ is neither sufficient nor necessary for the existence of a maximal set in a model. Proposition 1 (c) applied to Corollary 1 and Theorem 4 proves the corresponding result for $I \Sigma_{1}$.

The model $\mathscr{J}$ of Theorem 4 is an example of one which is reminiscent of an admissible ordinal $\alpha$ whose $\Sigma_{1}$ projectum is less than $\alpha$ (although these are also dissimilar in many respects). Bearing this in mind, it is not too difficult to see that if $\mathscr{M}$ is a model of some fragment of Peano arithmetic (at least as strong as $P^{-}+I \Sigma_{0}$ ) with a $\Delta_{2}(\mathscr{M})$ map from $\mathscr{N}$ onto $\mathscr{M}$, then there is a maximal set in $\mathscr{M}$ (cf. Lerman and Simpson [7] for the admissible case).

Note added in proof. T. Slaman has pointed out that the construction in Theorem 2 allows one to argue the stronger conclusion that $P^{-}+I \Sigma_{2}$ implies the cxistence of a maximal set. This follows from the fact that in a model of $P^{-}+I \Sigma_{2}$, the crucial lemma for infinite injury constructions, that the truth path in a tree method constrution is the left most path visited infinitely often, holds. In our situation, this trans- 
lates into the observation that if $M$ is not maximal, then there is a least $e$-state which receives unboundedly many new elements. A finite injury argument shows that this is not possible.

\section{REFERENCES}

[1] C. T. Chong, Techniques of Admissible Recursion Theory, Lecture Notes in Math. 1106, Springer Verlag, 1984.

[2] C. T. Chong and M. Lerman, Hyperhypersimple $\alpha$-r.e. sets, Ann. of Math., Logic, 9 (1976), 1-48.

[ 3 ] R. M. Friedberg, Three theorems on recursive enumeration: I. Decomposition, II. Maximal set, III. Enumeration without duplication, J. Symbolic Logic, 23 (1958), 309-316.

[4] M. J. Groszek and M. E. Mytilinaios, $\Sigma_{2}$ induction and the construction of a high degree, to appear.

[5] M. J. Groszek and T. A. Slaman, Foundations of the priority method I: Finite and infinite injury, to appear.

[6 ] L. A. Kirby and J. B. Paris, $\Sigma_{n}$ collection schemas in arithmetic, in: Logic colloquium '77, North-Holland, 1978, 199-209.

[ 7 ] M. Lerman and S. J. Simpson, Maximal sets in $\alpha$-recursion theory, Israel J. Math., 4 (1973), 236-247.

[8] M. E. Mytilinaios, Finite injury and $\Sigma_{1}$ induction, J. Symbolic Logic, 54 (1989), $38-49$.

[9] M. E. Mytilinaios and T. A. Slaman, $\Sigma_{2}$ collection and the infinite injury priority method, J. Symbolic Logic, 53 (1988), 212-221.

[10] T. A. Slaman and W. H. Woodin, $\Sigma_{1}$ collection and the finite injury priority method, to appear.

[11] R. A. Shore, On the jump of an $\alpha$-recursively enumerable set, Trans. Amer. Math. Soc., 217 (1976), 351-363.

[12] R. I. Soare, Recursively Enumerable Sets and Degrees, $\Omega$ Series, Springer Verlag, 1987.

Department of Mathematics

National University of Singapore

Kent Ridge, Singapore 0511

Republic of Singapore 